\title{
Technical Assessment of Spirometers Connected in Series
}

\author{
Quentin Lefebvre, Thomas Vandergoten, Eric Derom MD PhD, \\ Emilie Marchandise $\mathrm{PhD}$, and Giuseppe Liistro MD PhD
}

\begin{abstract}
BACKGROUND: Office spirometers are now widely used to detect obstructive lung diseases. To test the technical characteristics of these devices, simulation of different forced expiratory maneuvers is performed, using computer generated waveforms. However, the tests with human subjects are also useful to detect technical flaws. The procedure used by some authors to test the accuracy of office spirometers is to compare measurements made by 2 spirometers connected in series. OBJECTIVE: The aim of this study was to evaluate the accuracy of this latter procedure. METHODS: Two sets of 2 spirometers connected in series were used: the PocketSpiro with the MicroLoop, and the PocketSpiro with the SpiroScout. Different standard American Thoracic Society curves were selected for both ambient temperature and pressure (ATP) and body temperature and pressure saturated (BTPS) conditions and generated with a waveform simulator. We compared lung function indices (FVC, peak expiratory flow, and $\mathrm{FEV}_{1}$ ) recorded by the PocketSpiro when it was placed respectively upstream or downstream in the assembly. RESULTS: In ATP conditions, lung function indices were generally higher when the spirometer was placed downstream rather than upstream. The observed deviations reached up to $10 \%$. In BTPS conditions, lung function indices were underestimated when the spirometer was placed downstream, as compared to the ATP procedure. The modification of the flow characteristics and the temperature drop are the $\mathbf{2}$ mechanisms that could explain our results. CONCLUSIONS: Connecting the spirometers in series gives variable results, depending on the position of the spirometer in the assembly. Individual tests are therefore essential, as results are not interchangeable. Key words: spirometry; instrumentation; quality control; COPD; respiratory function tests; technology assessment; benchmarking. [Respir Care 2012; 57(8):1273-1277. (C) 2012 Daedalus Enterprises]
\end{abstract}

\section{Introduction}

Obstructive lung diseases such as COPD are one of the leading causes of mortality in the world. ${ }^{1}$ The diagnosis of COPD should be confirmed by the measurement of $\mathrm{FEV}_{1}$, $\mathrm{FVC}$, and $\mathrm{FEV}_{1} / \mathrm{FVC},{ }^{1}$ for which a spirometer is needed. Thanks to the improvement of micro-computers, a growing number of spirometers are currently in development

\footnotetext{
Mr Lefebvre, Mr Vandergoten, and Dr Marchandise are affiliated with the Institute of Mechanics, Materials and Civil Engineering, Université Catholique de Louvain, Louvain-la-Neuve, Belgium. Dr Derom is affiliated with the Department of Respiratory Medicine, Ghent University Hospital, Ghent, Belgium. Dr Liistro is affiliated with the Pneumology Department, Cliniques Universitaires Saint Luc, Université Catholique de Louvain, Brussels, Belgium.
}

The authors have disclosed a relationship with MEC (Medical Electronic Construction) Group. and marketed worldwide. Those devices, called handheld spirometers or office spirometers, are relatively cheap, can be connected to a desktop computer, and are thus well suited for general practitioners to perform routine spirometry that was previously the domain of pulmonary specialists. $^{2}$

To test those office spirometers, manufacturers generally use displacement pump systems to mechanically reproduce the American Thoracic Society (ATS) standard waveforms. ${ }^{3}$ Indeed, the ATS has published criteria describing adequate spirometer performances and suggested

\footnotetext{
Correspondence: Giuseppe Liistro MD PhD, Pneumology Department, Cliniques Universitaires Saint Luc, 10 Avenue Hippocrate, 1200 Brussels, Belgium. E-mail: giuseppe.liistro@uclouvain.be.
}

DOI: $10.4187 /$ respcare. 01464 
that these parameters should be verified before marketing the equipment. ${ }^{4}$ However, in order to validate spirometers for human subjects, some studies assessed spirometric accuracy by comparing one small spirometer with a conventional laboratory one, connecting those 2 devices in series. $^{5-9}$ This procedure avoids inter-maneuver variability and thus reduces the number of subjects and time required for testing to validate a spirometer. The aim of this study was to evaluate the validity of the testing procedure with the spirometers connected in series.

\section{Methods}

\section{Materials}

The study was carried out with a commercially available computer-controlled mechanical syringe (1120 flow/ volume generator, Hans Rudolph, Shawnee, Kansas). According to the manufacturer, this device is able to generate flows up to $16 \mathrm{~L} / \mathrm{s}$ and volumes up to $8.5 \mathrm{~L}$. It can perform any kind of previously sampled and recorded flow-time curve downloaded in the waveform editor of the HansRudolph simulator.

The flow-volume simulator was connected to the spirometers using a rubber connector of a total length of $100 \mathrm{~mm}$. However, once connected, the distance between the simulator and the spirometers was only $55 \mathrm{~mm}$. The internal and external diameters of the outlet of the simulator were $30 \mathrm{~mm}$ and $45 \mathrm{~mm}$, respectively.

Three different spirometers, presented in Table 1, were tested. They all comply with the recommendations of the ATS, according to their manufacturers. ${ }^{3}$ If connected to a desktop computer provided with the appropriate software (see Table 1), these devices make spirometry readily accessible to the general practitioner. They measure instantaneous flows that are integrated electronically into volumes, expressed in BTPS conditions. Only the SpiroScout and the MicroLoop require a volume calibration involving calibration checks or recalibration factors.

\section{Protocol}

A total of 4 combinations of 2 spirometers each were assembled: the PocketSpiro (placed upstream) with the MicroLoop (placed downstream) and vice-versa, and the PocketSpiro (placed upstream) with the SpiroScout (placed downstream) and vice versa (Figure). To connect the spirometers in series, we used 4 different cone-shaped rubber connectors. The connectors were thin $(1.2 \mathrm{~mm})$ and they were positioned inside the outlet of the upstream spirometer and outside the mouthpiece (or inlet) of the downstream spirometer.

\section{QUICK LOOK}

\section{Current knowledge}

Office spirometers are widely used to detect obstructive lung disease. Testing the technical characteristics of spirometers is accomplished using simulation of various forced expiratory maneuvers and computer generated waveforms.

\section{What this paper contributes to our knowledge}

Comparison of spirometers connected in series is an unacceptable method that gives inaccurate and variable results, depending on the position of the spirometer in the assembly. Individual testing remains the method of choice to evaluate the performance of new spirometers.

The length of the connectors was $50 \mathrm{~mm}$. The internal diameters of the outlets and external diameters of the inlets of the spirometers were as follows:

A: $24 \mathrm{~mm}$ internal diameter $\rightarrow 30.5 \mathrm{~mm}$ external diameter

B: $24 \mathrm{~mm}$ internal diameter $\rightarrow 22 \mathrm{~mm}$ external diameter

C: $33 \mathrm{~mm}$ internal diameter $\rightarrow 27 \mathrm{~mm}$ external diameter

D: $19.5 \mathrm{~mm}$ internal diameter $\rightarrow 27 \mathrm{~mm}$ external diameter

The letters A through D correspond to the settings described in Figure 1.

Three of the 24 ATS standard volume-time curves, numbers 1,17 , and 23 , and one of the 26 ATS flow-time curves, number 25 , were selected according to differences in shape and complexity. ${ }^{3}$

Each combination of spirometers was submitted to the 4 curves, each curve being delivered by the Hans Rudolph simulator 3 times, under both ambient temperature and pressure (ATP), and body temperature and ambient pressure saturated with water vapor (BTPS) conditions. To simulate real biological conditions as much as possible, we used an external humidifier to obtain $100 \%$ relative humidity, and the heater included as an option in the lung simulator to warm up the air to $37^{\circ} \mathrm{C}$.

The manufacturer's specifications and instructions were followed with the highest accuracy, in particular concerning the handling of the spirometers and their need for calibration checks before each series of tests.

\section{Statistical Analysis}

Three clinically relevant parameters were analyzed: peak expiratory flow (PEF), FVC, and $\mathrm{FEV}_{1}$. Since the aim of the study was to compare the measurements made by the same spirometer according to its position in the assembly, 
Table 1. Office Spirometers Characteristics

\begin{tabular}{|c|c|c|c|}
\hline Model & $\begin{array}{l}\text { Manufacturer, } \\
\text { Country }\end{array}$ & Flow Sensor & Software \\
\hline PocketSpiro & MEC, Belgium & $\begin{array}{l}\text { Pneumotachometer, } \\
\text { variable orifice } \\
\text { membrane }\end{array}$ & PDI \\
\hline MicroLoop & $\begin{array}{l}\text { Micro Medical, } \\
\text { England }\end{array}$ & Turbine & Spida5 \\
\hline SpiroScout & Ganshorn, Germany & Ultrasonic & LF8 \\
\hline
\end{tabular}

the absolute accuracy of the spirometers was not specifically assessed. For each assembly and each of the 2 test conditions we measured the deviation between the value given by a spirometer placed upstream and the value reported by the same spirometer placed downstream, using the following equation:

Deviation $(\%)=100 \times($ value upstream

- value downstream)/value upstream

\section{Results}

Table 2 presents the observed deviations expressed in percentage according to the equation. A negative deviation means that the value was higher when the spirometer was placed downstream rather than upstream in the assembly. We observed deviations up to $10 \%$ when testing under ATP or BTPS conditions for FVC, and up to $4 \%$ for FEV in ATP conditions. In BTPS conditions we found, mainly for the assembly PocketSpiro-MicroLoop, that the measurements of the spirometer placed upstream were lower, as compared to the ATP procedure.

Particularly in the case of the SpiroScout placed in series with the PocketSpiro, the deviations were often positive and higher for the measurement of FVC. Figure 1 shows the values of $\mathrm{FEV}_{1}$ measured by the PocketSpiro when it was connected in series with the 2 other devices using curve number 1 of the standard volume-time curves (target $\mathrm{FEV}_{1} 4.262 \mathrm{~L}$ ). There was a substantial difference in $\mathrm{FEV}_{1}$ when the PockectSpiro was placed after the MicroLoop.
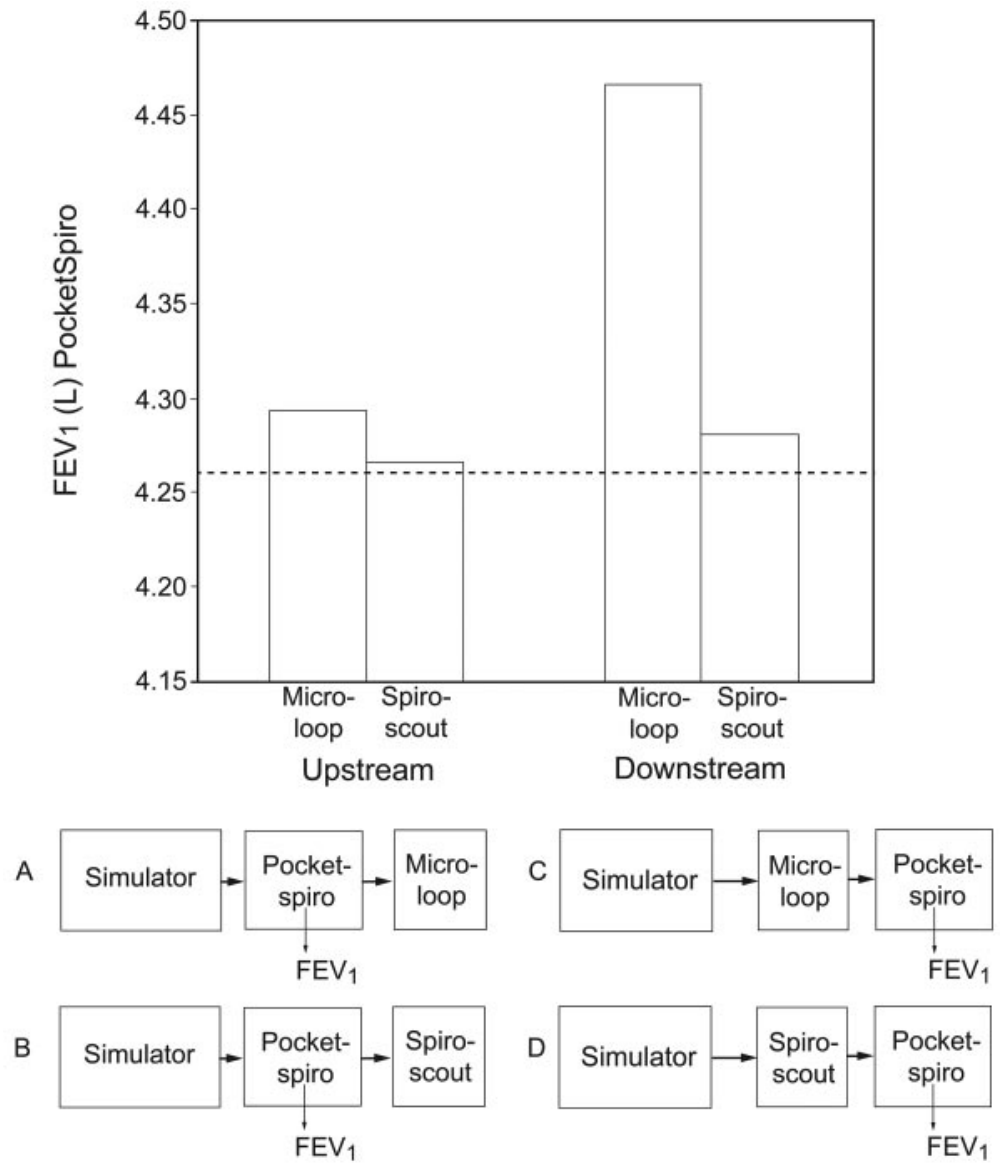

Figure. FEV 1 measured by the PocketSpiro placed (A) upstream of the MicroLoop, (B) upstream of the SpiroScout, (C) downstream of the MicroLoop, (D) downstream of the SpiroScout. The FEV 1 was measured using the American Thoracic Society curve 1/24, generated by the Hans-Rudolph simulator, under ambient temperature and pressure conditions, and the target value was $4.262 \mathrm{~L}$ (dashed line). 
Table 2. Deviations Between Measurements of FVC, $\mathrm{FEV}_{1}$, and PEF for 2 Sets of 2 Spirometers Each*

\begin{tabular}{|c|c|c|c|c|c|c|c|}
\hline & \multirow{2}{*}{ ATS Curve } & \multicolumn{2}{|c|}{$\mathrm{FVC}(\%)$} & \multicolumn{2}{|c|}{$\mathrm{FEV}_{1}(\%)$} & \multicolumn{2}{|c|}{ PEF $(\%)$} \\
\hline & & ATP & BTPS & ATP & BTPS & ATP & BTPS \\
\hline \multicolumn{8}{|c|}{ Set PocketSpiro - MicroLoop } \\
\hline \multirow[t]{4}{*}{ PocketSpiro } & $1 / 24$ & $-2.60 \dagger$ & -1.88 & $-4.04 \dagger$ & -1.96 & -1.98 & -0.69 \\
\hline & $17 / 24$ & 0.06 & -0.24 & -1.78 & -0.81 & -1.08 & -0.66 \\
\hline & $23 / 24$ & 0.94 & 1.41 & -0.48 & 0.51 & -1.15 & -1.41 \\
\hline & $25 / 26$ & -1.16 & -0.84 & -1.50 & -0.97 & -3.29 & -1.41 \\
\hline \multirow[t]{4}{*}{ MicroLoop } & $1 / 24$ & -0.77 & 0.84 & -0.61 & 0.47 & -0.65 & 0.60 \\
\hline & $17 / 24$ & -0.11 & 0.93 & -0.38 & 0.39 & -1.88 & 0.12 \\
\hline & $23 / 24$ & -0.39 & 0.69 & -0.24 & 1.22 & -0.82 & -1.37 \\
\hline & $25 / 26$ & -1.05 & 0.00 & -1.16 & 0.00 & -1.80 & -1.04 \\
\hline \multicolumn{8}{|c|}{ Set PocketSpiro - SpiroScout } \\
\hline \multirow[t]{4}{*}{ PocketSpiro } & $1 / 24$ & -0.33 & -1.20 & -0.39 & -1.28 & 0.76 & -0.83 \\
\hline & $17 / 24$ & -0.23 & -1.47 & -0.13 & -1.70 & -1.05 & -0.96 \\
\hline & $23 / 24$ & 0.19 & -0.59 & -0.24 & -1.23 & -0.21 & -3.41 \\
\hline & $25 / 26$ & 0.00 & 0.16 & 0.06 & 0.00 & -1.18 & 1.38 \\
\hline \multirow[t]{4}{*}{ SpiroScout } & $1 / 24$ & $3.15 \dagger$ & -1.15 & 1.88 & -0.24 & 0.10 & 0.31 \\
\hline & $17 / 24$ & $8.52 \dagger$ & $10.42 \dagger$ & 1.03 & 2.08 & 1.72 & 2.17 \\
\hline & $23 / 24$ & $10.97 \dagger$ & $-5.23 \dagger$ & 0.50 & -1.79 & -0.20 & -2.13 \\
\hline & $25 / 26$ & 1.36 & -0.43 & 0.63 & -0.41 & -0.19 & 1.64 \\
\hline
\end{tabular}

\footnotetext{
* Spirometers were connected in series using the sets: PocketSpiro - MicroLoop, and PocketSpiro - SpiroScout. Data represent the subtraction of the measurements of a given spirometer, placed upstream, and the same spirometer placed downstream within one set (see equation). Lung function values were recorded for 4 different American Thoracic Society (ATS) waveforms in ambient temperature and pressure (ATP) and body temperature and ambient pressure saturated with water vapor (BTPS) conditions. For example, with ATS curve 1/24, FVC measured by the PocketSpiro was 6.020 L upstream, and 6.176 L downstream with the MicroLoop. Following the equation, $(6.020-6.176) \times 100 / 6.020=-2.6 \%$.

$\dagger$ The absolute difference between the measurements obtained upstream and downstream was $>150 \mathrm{~mL}$.

$\mathrm{PEF}=$ peak expiratory flow
}

\section{Discussion}

Our data show that the position of the spirometer connected in series influences the results generated by the spirometer, with differences in magnitude that under certain circumstances and for some of the tested spirometers ranged between 5\% and 10\%. As shown in Table 2, these differences occasionally exceeded the recommended between-maneuver repeatability criteria for $\mathrm{FEV}_{1}$ and $\mathrm{FVC}$, which should remain below $150 \mathrm{~mL}$ for $\mathrm{FEV}_{1}$ and FVC. ${ }^{4}$

It seems difficult to identify a general trend in the deviations observed, which do not appear to be related to one particular curve, one of the spirometric variables, or the degree of humidity or temperature. Possibly a number of parameters, such as resistance, dead space volume, and curve dynamics, may affect the $\mathrm{FEV}_{1}$, FVC, or PEF value generated by the spirometer. Likewise, the deviations observed in ATP conditions cannot be explained by the drop in temperature and humidity, as could be expected in BTPS conditions. We thus tend to attribute the observed deviations to the change in flow characteristics produced by the connection in series of 2 spirometers. One possible explanation could be that the geometry of the upstream spirometer disrupts the flow in the downstream spirometer, which might then become turbulent. Under these circumstances, the linearization table of the device is no longer adequate and the measurements are distorted.

Surprisingly, Barr et al previously reported that connecting spirometers in series gave acceptable results. ${ }^{6}$ However, careful analysis of the Bland-Altman plot of the FVC published in that paper showed a clear proportional error, the difference between the EasyOne spirometer and the reference spirometer became increasingly more prominent with higher FVC. These authors were unable to reproduce that proportional error if the tests were not performed in series, but in standard conditions.

We also observed that the deviations obtained for the PocketSpiro were more pronounced when it was in series with the MicroLoop than with the SpiroScout (see Table 2 and Fig. 1). This finding can certainly be ascribed to the characteristics of some of the spirometers under investigation. Indeed, the MicroLoop uses a turbine (moving parts) in the flow sensor, whereas the SpiroScout uses an ultrasonic sensor (no moving parts) and therefore a lower resistance. Such differences may largely explain why the flow downstream from the SpiroScout was only slightly disturbed, compared to the MicroLoop.

We also found important differences between data obtained in BTPS and ATP conditions for the PocketSpiroMicroLoop assembly. This was particularly clear for spi- 


\section{Technical Assessment of Spirometers Connected in Series}

rometer placed upstream. This could possibly result from the drop in actual temperature between the simulator and the second spirometer, which could be larger than the expected value $\left(3-4^{\circ} \mathrm{C}\right)$ programmed in the software of that spirometer. The lower observed volume might thus be the consequence of an underestimated expiratory correction factor and an insufficient conversion of the measured volume into BTPS conditions.

\section{Conclusions}

We conclude that comparison of spirometers connected in series is an inappropriate and unacceptable method that gives inaccurate and even variable results, depending on the position of the spirometer under investigation in the assembly. Since the results are not interchangeable, when the devices are tested in human subjects, individual spirometer testing remains the method of choice to evaluate performance of new spirometers.

\section{ACKNOWLEDGMENTS}

We would like to thank Jean-Yves Moens, MEC (Medical Electronic Construction), Brussels, Belgium, for allowing us to use his Hans Rudolph flow/volume simulator to conduct our tests.

\section{REFERENCES}

1. Global Initiative for Chronic Obstructive Lung Disease (GOLD). Global strategy for the diagnosis, management, and prevention of chronic obstructive pulmonary disease. Updated 2011. http:// www.goldcopd.org/uploads/users/files/GOLD_Report_2011)Feb21. pdf. Accessed May 22, 2012.

2. Buffels J, Degryse J, Heyrman J, Decramer M. Office spirometry significantly improves early detection of COPD in general practice: the DIDASCO Study. Chest 2004;125(4):1394-1399.

3. American Thoracic Society. Standardization of spirometry, 1994 update. Am J Respir Crit Care Med 1995;152(3):1107-1136.

4. Miller MR, Hankinson J, Brusasco V, Burgos F, Casaburi R, Coates A, et al. Standardisation of spirometry. Eur Respir J 2005;26(2): 319-338.

5. Abboud S, Bruderman I. Assessment of a new transtelephonic portable spirometer. Thorax 1996;51(4):407-410.

6. Barr RG, Stemple KJ, Mesia-Vela S, Basner RC, Derk SJ, Henneberger PK, et al. Reproducibility and validity of a handheld spirometer. Respir Care 2008;53(4):433-441.

7. Bastian-Lee Y, Chavasse R, Richter H, Seddon P. Assessment of a low-cost home monitoring spirometer for children. Pediatr Pulmonol 2002;33(5):388-394.

8. Chowienczyk PJ, Lawson CP. Pocket-sized device for measuring forced expiratory volume in one second and forced vital capacity. Br Med J (Clin Res Ed) 1982;285(6334):15-17.

9. Mortimer KM, Fallot A, Balmes JR, Tager IB. Evaluating the use of a portable spirometer in a study of pediatric asthma. Chest 2003; 123(6): 1899-1907. 